\title{
What BPM Technology Can Do for Healthcare Process Support
}

\author{
Manfred Reichert \\ Institute of Databases and Information Systems, Ulm University, Germany \\ manfred.reichert@uni-ulm.de
}

\begin{abstract}
Healthcare organizations are facing the challenge of delivering personalized services to their patients in a cost-effective and efficient manner. This, in turn, requires advanced IT support for healthcare processes covering both organizational procedures and knowledge-intensive, dynamic treatment processes. Nowadays, required agility is often hindered by a lack of flexibility in hospital information systems. To overcome this inflexibility a new generation of information systems, denoted as process-aware information systems (PAISs), has emerged. In contrast to data- and function-centered information systems, a PAIS separates process logic from application code and thus provides an additional architectural layer. However, the introduction of process-aware hospital information systems must neither result in rigidity nor restrict staff members in their daily work. This keynote presentation reflects on recent developments from the business process management (BPM) domain, which enable process adaptation, process flexibility, and process evolution. These key features will be illustrated along existing BPM frameworks. Altogether, emerging BPM methods, concepts and technologies will contribute to further enhance IT support for healthcare processes.
\end{abstract}

\section{Introduction}

Business process support has been a major driver for enterprise information systems for a long time. Its overall goal is to overcome the drawbacks of functional over-specialization and lack of process control. Technology response to this business demand was met with a suite of technologies ranging from office automation tools to workflow systems to business process management technology.

Just as database management systems provide a means of abstracting application logic from physical data aspects, workflow management systems separate coordinative process logic from application code [1, 2]. Although workflow technology has delivered a great deal of productivity improvements in industry, it has been mainly designed for the support of pre-specified and repetitive business processes requiring a basic level of coordination between human performers and required application services. More recently business process management (BPM) has been used as broader term to reflect the fact that business processes may or may not involve human participants, cross organizational boundaries, and require a high degree of flexibility $3,4,4]$. 
Currently, there is a widespread interest in BPM technologies in a variety of domains, especially in the light of emerging software paradigms surrounding service-oriented computing and its application to dynamic service orchestration and choreography. In this context, the notion of PAIS (Process Aware Information System) provides a guiding framework to understand and deliberate on the above developments [5]. As fundamental characteristic, a PAIS provides the basic means to separate process logic from application code. Furthermore, it has to cover all phases of the process lifecycle; i.e., process design, process implementation, process configuration, process enactment, process monitoring and diagnosis, and process evolution [6]. At build-time the process logic has to be explicitly defined based on the constructs provided by a process modeling language. In this context, a variety of workflow patterns (e.g., control and data patterns, resource patterns, time patterns) have been suggested enabling the comparison and evaluation of existing modeling languages and tools $7-10]$. Other works, in turn, target at improved process model quality and understandability 11 13. At run-time a PAIS executes the processes according to the defined logic (i.e., process model) and orchestrates required application services and other resources. Examples of PAIS-enabling technologies include process management systems like ADEPT2 [14], AristaFlow [15], and YAWL [16] as well as case handling frameworks like FLOWer [4] and PHILHarmonic Flows [17].

In spite of several success stories on the uptake of PAISs in industry and the growing process-orientation of enterprises, BPM technologies have not had the widespread adoption in the healthcare domain yet [18]. A major reason for this has been the rigidity enforced by first generation workflow management systems, which inhibits the ability of a hospital to respond to process changes and exceptional situations in an agile way [19]. When efforts are taken to improve and automate the flow of healthcare processes, however, it is extremely important not to restrict physicians and nurses. Early attempts to change the functionoriented views of patient processes have been unsuccessful whenever rigidity came with them. Variations in the course of a disease or a treatment process are deeply inherent to medicine, and the unforeseen event is to some degree a "normal" phenomenon. Therefore, healthcare process support craves for advanced BPM technologies enabling flexible, adaptive and evolutionary processes in large scale.

For many years the BPM community has recognized that a PAIS needs to be able to cope with real-world exceptions, uncertainty, and evolving processes [20]. To address respective needs a variety of process support paradigms like adaptive processes, case handling, and constraint-based processes have been suggested and been applied in practice. Basically, these paradigms allow for coordinated process support (involving human actors) and application service orchestration on the one hand, while enabling process flexibility, process adaptation, and process evolution on the other hand. For example, adaptive PAISs allow dynamically changing the process model of an ongoing process instance during run-time [21], while constraint-based paradigms enable loosely specified process models, which can be dynamically refined at the process instance level obeying pre-defined 
constraints 22]. Taking these technological developments from the BPM area, new perspectives for the realization of flexible, process-aware hospital information systems emerge.

This keynote presentation elaborates on advanced BPM methods, concepts and technologies developed during the last decade. Emphasis is put on key features enabling process flexibility, process adaptation, and process evolution. Based on them advanced PAIS can be realized being able to flexibly cope with real-world exceptions, uncertainty, and change. Respective methods, concepts and technologies will foster the development of a new generation of processaware healthcare information systems.

Section 2 draws a realistic picture of the environment in which a process-aware hospital information systems must run. Section 3 discusses core contributions from the BPM domain enabling flexible and dynamic process support. Finally, the paper concludes with a short summary and outlook in Section 4.

\section{Hospital Working Environment}

This section motivates the need for healthcare process support and discusses the conditions under which a process-aware hospital information system must operate 18, 19].

Generally, in hospitals, the work of physicians and nurses is burdened by numerous organizational as well as medical tasks. Medical procedures must be planned and prepared, appointments be made, and results be obtained and evaluated. Usually, in the diagnostic and treatment process of a particular patient various, organizationally more or less separate units are involved. For a patient treated in a department of internal medicine or surgery, for example, tests and procedures at the laboratory and the radiology department may become necessary. In addition, specimen or the patients themselves have to be transported, physicians from other units may need to come and see the patient, and reports have to be written, sent, and evaluated. Thus, the cooperation between organizational units as well as the medical staff is a vital task with repetitive, but nevertheless non-trivial character. Processes of different complexity and duration can be identified. One can find short organizational procedures like order entry and result reporting for radiology, but also complex and long-running (even cyclic) treatment processes like chemotherapy for in- and out-patients.

Physicians have to decide which interventions are necessary or not - under the perspective of costs and invasiveness - or which are even dangerous because of possible side-effects or interactions. Many procedures need preparatory measures of various, sometimes considerable complexity. Before a surgery can take place, for example, a patient has to undergo numerous preliminary examinations, each of them requiring additional preparations. While some of them are known in advance, others may have to be scheduled dynamically, depending on the individual patient and her state of health. All tasks may have to be performed in certain orders, sometimes with a certain time distance between them. After an injection with contrast medium was given to a patient, for example, some other tests 
cannot be performed within a certain period of time. Usually, physicians have to coordinate the tasks related to their patients manually, taking into account all the dependencies existing between them. Changing a schedule is not trivial and requires time-consuming communication. For some procedures, physicians from various departments have to work together; i.e., coherent series of appointments have to be arranged and for each step actual and adequate information has to be provided. Typically, each unit involved in the treatment process concentrates on the function it has to perform. Thus, the process is subdivided into partial, function- and organization-oriented views, and optimization usually stops at the border of the department. For all these reasons many problems result:

- Patients have to wait, because resources (e.g., physicians, rooms or technical equipment) are not available.

- Medical procedures may become impossible to perform, if information is missing, preparations have been omitted, or a preceding procedure has been postponed, canceled or requires latency time. Depending procedures may then have to be re-scheduled as well resulting in numerous phone-calls and time losses.

- If any results are missing but urgently needed, tests or procedures may have to be performed repeatedly.

Because of this, from the patient as well as from the hospital perspective unpleasant and undesired effects occur: Hospital stays can be longer than necessary and the costs or even the invasiveness of the patient treatment may increase. In critical situations, missing information may lead to late or even wrong decisions. Investigations have shown that medical personnel is aware of these problems and that computer systems helping to make appointments and providing the necessary information would be highly welcome by nurses and physicians. In an increasing way it is being understood that correlation between medicine, organization and information is high, and that current organizational structures and hospital information systems offer sub-optimal support. This is even more the case for hospital-wide and cross-hospital processes and for health care networks.

The roles of physicians and nurses complicate the problem. They are responsible for many patients and they have to provide an optimal treatment process for each of them. Medical tasks are critical to patient care and even minor errors may have disastrous consequences. The working situation is further burdened by frequent context switches. Physicians often work at various sites of a hospital in different roles. In many cases unforeseen events and emergency situations occur, patient status changes, or information necessary to react is missing (up to: "where is my patient?"). In addition, the physician is confronted with a massive load of data to be structured, intellectually processed, and put into relation to the problems of the individual patient. Typically, physicians tend to make mistakes (e.g., wrong decisions, omissive errors) under this data overload.

From the perspective of a patient, a concentration on his treatment process is highly desirable. Similarly, medical staff members wish to treat and help patients and not to spend their time on organizational tasks. From the perspective 
of health care providers, the huge potential of the improvement of healthcare processes has been identified: length of stay, number of procedures, and number of complications could be reduced. Hence there is a growing interest in process orientation and quality management. Medical and organizational processes are being analyzed, and the role of medical guidelines describing diagnostic and treatment steps for given diagnoses is emphasized 23 25].

\section{$3 \quad$ IT Support for Healthcare Processes}

\subsection{Flexibility Demands of Healthcare Processes}

Obviously, the IT support for healthcare processes must not introduce rigidity or restrict medical staff members in their daily work. Generally, physicians and nurses must be free to react and are trained to do so. In an emergency case, for example, physicians may collect information about a patient by phone and proceed with the treatment process, without waiting for the electronic report to be written. Furthermore, medical procedures may have to be aborted if the patient's health state gets worse or the provider finds out that a prerequisite is not met. Such deviations from the pre-planned process are frequent and form a key part of process flexibility in hospitals. Any computer-based system which is used to assist physicians and nurses in their daily work, therefore, must allow them to gain complete initiative whenever needed. In particular, process-aware hospital information systems must be easy to handle, self-explaining, and - most important - their use in exceptional situations must be not more cumbersome and time-consuming than simply handling the exception by a telephone call to the right person. Otherwise the PAIS will not be accepted by hospital staff.

In summary, process-aware hospital information system must be able to cope with exceptions, uncertainty, and evolving processes.

\subsection{Pre-specified vs. Loosely Specified Process Models}

In the predominant process support paradigm, PAISs require the a priori specification of all process details. The resulting pre-specified process models are then used as schema for process execution. Typically, such a pre-specified process model defines all activities to be executed, their control flow and data flow dependencies, organizational entities performing the activities, the data objects manipulated by them, and the application services invoked during their execution. In this context, a variety of modeling patterns has been suggested [-10], which can be used as building blocks for creating process models and which allow adding some build-in-flexibility to these models (i.e., flexibility-by-design).

However, IT support for healthcare processes demands a more agile approach recognizing the fact that in dynamic environments pre-specified processes are outdated fast and thus require closer interweaving of modeling and execution. Consequently, any PAIS relying on pre-specified process models not only needs to be able to adequately cope with real-world exceptions [26], to adapt the execution of single process instances (i.e., business cases) on-the-fly [27], to efficiently deal 
with uncertainty [20], and to cope with variability 28], but must also support the evolution of business processes over time, e.g., due to changing regulations or organizational changes [29, 30]. Respective features are provided by adaptive PAISs 21, 27] that have emerged in the BPM area during the last years.

In addition to pre-specified processes, which provide a reliable schema for process execution and thus are well suited for automating repetitive and rather predictable processes, existing approaches also allow process designers to only provide a loosely specified process model [31, 32] which can then be refined by endusers during run-time taking predefined constraints into account (e.g., mutual exclusion of two activities or activity orders to be obeyed).

In practice, there also exist processes that can neither be adequately captured in pre-specified models nor in constraint-based ones. In particular, it has been recognized that knowledge-intensive processes (e.g., treatment processes) cannot always be "straight-jacketed" into activities. Prescribing an activity-centric process model for them would lead to a "contradiction between the way processes can be modeled and the preferred work practice" 33. As shown in the context of the PHILharmonicFlows project a major reason for this deficiency stems from the unsatisfactory integration of processes and data in existing activitycentric PAISs [34, 35]. To remedy this deficiency, [36] analyzed various processes from different domains which are not adequately supported by existing PAIS. As a major insight it was found out that in many cases process support requires object-awareness. In particular, process support has to consider object behavior as well as object interactions, and should therefore be based on two levels of granularity. Besides this, object-awareness requires data-driven process execution and integrated access to processes and data. PHILharmonicFlows has identified basic properties of object-aware processes as well as fundamental requirements for their operational support. The developed PHILharmonicFlows framework 17] addresses these requirements and enables object-aware process management in a comprehensive manner distinguishing between micro and macro processes. For example, a micro process may coordinate the processing of a particular medical order, whereas a macro process may comprise all micro processes related to a particular entity (e.g., patient) as well as their coordination dependencies.

\subsection{Dealing with Exceptions, Uncertainty and Evolving Processes}

For more than a decade the BPM community has recognized that PAISs need to provide different kinds of build- and run-time flexibility [20]. Consequently, a variety of techniques have been developed ranging from fully pre-specified processes with certain built-in flexibility to ad-hoc adaptations of pre-specified processes during their execution to loosely specified processes (that have to be concretized during run-time). Generally, existing approaches can be characterized along three fundamental dimensions, namely IT support for adaptation, flexibility, and evolution:

- Adaptation represents the ability of the implemented processes to cope with exceptional circumstances [27]. On the one hand, existing PAISs like 
YAWL [16] provide support for the handling of expected exceptions, which can be anticipated and thus be pre-specified in the process model. Complementaty to this, adaptive PAISs like ADEPT2 37] enable the handling of unanticipated exceptions, e.g., through structural ad-hoc adaptations of single process instances (e.g., by adding, deleting or moving process activities during run-time). Thereby, users are assisted in defining ad-hoc adaptations and in reusing knowledge gathered in similar problem context in the past [6, 38]. Clearly, dynamic process adaptations necessitate a comprehensive framework ensuring correctness and robustness of the PAIS. The ADEPT2 framework, for example, provides sophisticated methods, concepts and tools for achieving these goals. In particular, ADEPT2 enables instance-specific changes of a pre-specified model in a controlled, correct and secure manner [27, 39]. This, in turn, can be considered as fundamental driver enabling flexible and dynamic processes in complex application environments. Note that when providing support for ad-hoc adaptations users are no longer forced to bypass the PAIS when unplanned exceptions occur. Instead, single processes instances can be dynamically adapted to the real-world situation if required. Finally, deviations from the pre-specified model are documented in respective change logs [40].

- Flexibility represents the ability of a process to execute on the basis of a loosely or partially specified model which is completed at run-time and may be unique to each process instance [31, 32]. Due to the high number of choices, not all of which can be anticipated and hence be pre-specified in a process model, frameworks like DECLARE [31] and Alaska [1] allow defining process models in a more relaxed manner; the model can be defined in a way that allows individual instances to determine their own (unique) processes. In particular, declarative approaches allow for loosely specified process models by following a constraint-based approach. While pre-specified process models define exactly how the overall task has to be accomplished, constraint-based process models focus on what should be done by describing the set of activities that may be performed as well as the constraints prohibiting undesired process behavior. Therefore, constraint-based approaches provide more build-in flexibility when compared to completely pre-specified process models. Potential advantages include the absence of over-specification and the provision of more maneuvering room for end-users. Generally, loosely specified models raise a number of challenges including the flexible configuration of process models at design time or their constraint-based definition during runtime. Due to the high number of run-time choices, in addition, more sophisticated user support (e.g., recommender systems) becomes necessary when compared to PAIS relying on fully pre-specified models. Finally, the integration of pre-specified and constraint-based processes constitutes an emerging area which is particularly important for the healthcare domain. In particular, well established criteria are needed to be able to decide which approach to take in which scenario and how to combine the two paradigms in the best possible way. 
- Evolution represents the ability of a process implemented in a PAIS to change when the business process evolves, e.g., due to legal changes or process optimizations [30, 42]. The assumption is that the processes have prespecified models, and a change causes these models to be modified. The biggest challenge then is the handling of the potentially large number of longrunning process instances, which were initiated based on the old model but are required to comply with the new specification from now on. Approaches like WASA2, ADEPT2 and WIDE allow process engineers to migrate such process instances to the new model version, while ensuring PAIS robustness and process consistency (see [43, 44]). Moreover, pre-specified process models often have to be changed to cope with model design errors, technical problems or poor model quality. In the latter context process model refactorings have been suggested to foster internal process model quality and to ensure maintainability of the PAIS over time [11, 45].

\subsection{Dynamic Processes and Process Learning}

In practice, there often exists a significant gap between what is prescribed in a model and what actually happens. Generally, a PAIS records the actual execution behavior of a collection of process instances in an execution log. Furthermore, adaptive PAISs document deviations from pre-specified models in change logs. In this context process mining strives to deliver a concise assessment of the organizational reality by mining these logs of dynamic processes [46]. Process discovery algorithms, for example, analyze execution logs and derive process models from them reflecting the actual process behavior best. Conformance testing, in turn, analyzes and measures discrepancies between the original model of a process and the actual execution of its instances (as recorded in execution logs). Finally, log-based verification checks the execution log for conformance with desired or undesired properties; e.g., process instance compliance with corporate guidelines or global regulations. Furthermore, change mining not only considers execution logs of process instances, but additionally analyzes the structural changes applied during the execution of process instances; i.e., they allow visualizing and analyzing dynamic deviations from pre-specified processes [47]. Finally, process variants mining [48, 49] allows discovering an optimal reference process model being "close" to a given collection of process variants; e.g., process instances derived from the same model, but structurally differing due to ad-hoc changes applied to them.

\section{Summary and Outlook}

When targeting IT support for healthcare processes it is important to distinguish the patient-specific medical treatment processes from the organizational procedures (e.g. order handling and result reporting) that generally coordinate the cooperation between staff members and organizational units within a hospital [18]. While the former are knowledge-intensive and highly dynamic 
processes, the latter constitute repetitive processes that capture the organizational knowledge necessary to coordinate daily tasks among staff members and between organizational units (e.g., wards and medical departments). Basically, BPM provides methods, concepts and tools for supporting both categories of processes. In particular, the described technological developments will allow providing the required process dynamics and flexibility, and thus foster the realization of a new generation of process-aware hospital information systems.

Still there is a gap between technology-driven approaches developed by the BPM community and methodological-based approaches suggested in the medical informatics field (e.g., clinical guideline support). Besides there still exists a number of challenges to be tackled in order to provide support for both organizational procedures and complex patient treatment processes. Amongst others these challenges include the process-oriented integration of heterogeneous systems, the embedding of IT process support into routine work practice, the learning from past process executions, the evolution of process knowledge over time, the real-time tracking of healthcare processes, and the coordination of interrelated processes (e.g., corresponding to the same patient). These issues are unlikely to be solved in near future, but indicate that interdisciplinary research is needed to further enhance IT support for healthcare processes.

\section{References}

1. Reijers, H.A., van der Aalst, W.M.P.: The effectiveness of workflow management systems: Predictions and lessons learned. Int'l Journal of Inf. Mgmt., 457-471 (2005)

2. Mutschler, B., Weber, B., Reichert, M.: Workflow management versus case handling - results from a controlled software experiment. In: Avanzi, R.M., Keliher, L., Sica, F. (eds.) SAC 2008. LNCS, vol. 5381, pp. 82-89. Springer, Heidelberg (2009)

3. Weske, M.: Business Process Management: Concepts, Methods, Technology. Springer, Heidelberg (2007)

4. Weber, B., Mutschler, B., Reichert, M.: Investigating the effort of using business process management technology: Results from a controlled experiment. Science of Computer Programming 75, 292-310 (2010)

5. Dumas, M., ter Hofstede, A.H.M., van der Aalst, W.M.P. (eds.): Process Aware Information Systems. Wiley Publishing, Chichester (2005)

6. Weber, B., Reichert, M., Wild, W., Rinderle-Ma, S.: Providing integrated life cycle support in process-aware information systems. Int'l J of Cooperative Information Systems 18, 115-165 (2009)

7. Lanz, A., Weber, B., Reichert, M.: Workflow time patterns for process-aware information systems. In: Bider, I., Halpin, T., Krogstie, J., Nurcan, S., Proper, E., Schmidt, R., Ukor, R. (eds.) BPMDS 2010 and EMMSAD 2010. LNBIP, vol. 50, pp. 94-107. Springer, Heidelberg (2010)

8. Russell, N., ter Hofstede, A., van der Aalst, W., Mulyar, N.: Workflow control-flow patterns: A revised view. Technical Report BPM-06-22, BPMcenter.org (2006)

9. Russell, N., van der Aalst, W.M.P., ter Hofstede, A.H.M.: Exception handling patterns in process-aware information systems. In: Martinez, F.H., Pohl, K. (eds.) CAiSE 2006. LNCS, vol. 4001, pp. 288-302. Springer, Heidelberg (2006) 
10. Weber, B., Rinderle, S., Reichert, M.: Change patterns and change support features in process-aware information systems. In: Krogstie, J., Opdahl, A.L., Sindre, G. (eds.) CAiSE 2007 and WES 2007. LNCS, vol. 4495, pp. 574-588. Springer, Heidelberg (2007)

11. Weber, B., Reichert, M., Reijers, H., Mendling, J.: Refactoring large process model repositories. Computers and Industry (2011)

12. Reijers, H., Mendling, J.: Modularity in process models: Review and effects. In: Dumas, M., Reichert, M., Shan, M.-C. (eds.) BPM 2008. LNCS, vol. 5240, pp. 20-35. Springer, Heidelberg (2008)

13. Mendling, J., Reijers, H.A., van der Aalst, W.M.P.: Seven process modeling guidelines (7PMG). Information and Software Technology 52, 127-136 (2009)

14. Reichert, M., Rinderle, S., Kreher, U., Dadam, P.: Adaptive process management with ADEPT2. In: Proc. ICDE 2005, pp. 1113-1114 (2005)

15. Reichert, M., et al.: Enabling Poka-Yoke workflows with the AristaFlow BPM Suite. In: Proc. BPM 2009 Demonstration Track. CEUR Workshop Proceedings, vol. 489 (2009)

16. ter Hofstede, A.H.M., van der Aalst, W.M.P., Adams, M., Russell, N.: Modern Business Process Automation: YAWL and Its Support Environment. Springer, Heidelberg (2009)

17. Künzle, V., Reichert, M.: PHILharmonicFlows: towards a framework for objectaware process management. Journal of Software Maintenance and Evolution: Research and Practice (2011)

18. Lenz, R., Reichert, M.: IT support for healthcare processes - premises, challenges, perspectives. Data and Knowledge Engineering 61, 39-58 (2007)

19. Dadam, P., Reichert, M., Kuhn, K.: Clinical workflows - the killer application for process-oriented information systems? In: Proc. BIS 2000, pp. 36-59 (2000)

20. Weber, B., Sadiq, S., Reichert, M.: Beyond rigidity - dynamic process lifecycle support: A survey on dynamic changes in process-aware information systems. Computer Science - Research and Development 23, 47-65 (2009)

21. Reichert, M., Rinderle-Ma, S., Dadam, P.: Flexibility in process-aware information systems. In: Jensen, K., van der Aalst, W.M.P. (eds.) Transactions on Petri Nets and Other Models of Concurrency II. LNCS, vol. 5460, pp. 115-135. Springer, Heidelberg (2009)

22. Weber, B., Reijers, H.A., Zugal, S., Wild, W.: The declarative approach to business process execution: An empirical test. In: van Eck, P., Gordijn, J., Wieringa, R. (eds.) CAiSE 2009. LNCS, vol. 5565, pp. 470-485. Springer, Heidelberg (2009)

23. Lenz, R., Blaser, R., Beyer, M., Heger, O., Biber, C., Baumlein, M., Schnabel, M.: IT support for clinical pathways-lessons learned. Intl. J. Med. Inform. 76, 397-402 (2007)

24. Peleg, M., Tu, S.W.: Design patterns for clinical guidelines. Intl. J. Med. Inform. 47, 1-24 (2009)

25. Peleg, M., Keren, S., Denekamp, Y.: Mapping computerized clinical guidelines to electronic medical records: Knowledge-data ontological mapper (KDOM). J. Biomed. Inform. 41, 180-201 (2008)

26. Reichert, M., Dadam, P., Bauer, T.: Dealing with forward and backward jumps in workflow management systems. Software and System Modeling 1, 37-58 (2003)

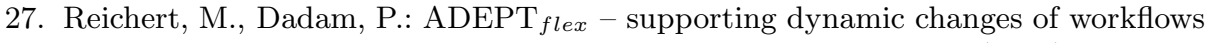
without losing control. Journal of Intelligent Inf. Sys. 10, 93-129 (1998)

28. Hallerbach, A., Bauer, T., Reichert, M.: Capturing variability in business process models: The Provop approach. Journal of Software Maintenance and Evolution: Research and Practice 22, 519-546 (2010) 
29. Reichert, M., Rinderle, S., Dadam, P.: On the common support of workflow type and instance changes under correctness constraints. In: Chung, S., Schmidt, D.C. (eds.) CoopIS 2003, DOA 2003, and ODBASE 2003. LNCS, vol. 2888, pp. 407-425. Springer, Heidelberg (2003)

30. Rinderle, S., Reichert, M., Dadam, P.: Flexible support of team processes by adaptive workflow systems. Distributed and Parallel Databases 16, 91-116 (2004)

31. Pesic, M.: Constraint-Based Workflow Management Systems: Shifting Control to Users. PhD thesis, Eindhoven University of Technology (2008)

32. Sadiq, S., Sadiq, W., Orlowska, M.: A framework for constraint specification and validation in flexible workflows. Information Systems 30, 349-378 (2005)

33. Sadiq, S., Orlowska, M.: On capturing exceptions in workflow process models. In: Proc. BIS 2000 (2000)

34. Künzle, V., Reichert, M.: Integrating users in object-aware process management systems: Issues and challenges. In: Rinderle-Ma, S., Sadiq, S., Leymann, F. (eds.) BPM 2009. LNBIP, vol. 43, pp. 29-41. Springer, Heidelberg (2010)

35. Künzle, V., Reichert, M.: Towards object-aware process management systems: Issues, challenges, benefits. In: Halpin, T., Krogstie, J., Nurcan, S., Proper, E., Schmidt, R., Soffer, P., Ukor, R. (eds.) Enterprise, Business-Process and Information Systems Modeling. LNBIP, vol. 29, pp. 197-210. Springer, Heidelberg (2009)

36. Künzle, V., Weber, B., Reichert, M.: Object-aware business processes: Fundamental requirements and their support in existing approaches. Int'l Journal of Information System Modeling and Design 2, 19-46 (2011)

37. Dadam, P., Reichert, M.: The ADEPT project: A decade of research and development for robust and flexible process support - challenges and achievements. Computer Science - Research and Development 23, 81-97 (2009)

38. Rinderle, S., Weber, B., Reichert, M., Wild, W.: Integrating process learning and process evolution - A semantics based approach. In: van der Aalst, W.M.P., Benatallah, B., Casati, F., Curbera, F. (eds.) BPM 2005. LNCS, vol. 3649, pp. 252-267. Springer, Heidelberg (2005)

39. Weber, B., Reichert, M., Wild, W., Rinderle, S.: Balancing flexibility and security in adaptive process management systems. In: Proc. CoopIS 2005, pp. 59-76 (2005)

40. Rinderle, S., Reichert, M., Jurisch, M., Kreher, U.: On representing, purging, and utilizing change logs in process management systems. In: Dustdar, S., Fiadeiro, J.L., Sheth, A.P. (eds.) BPM 2006. LNCS, vol. 4102, pp. 241-256. Springer, Heidelberg (2006)

41. Weber, B., Pinggera, J., Zugal, S., Wild, W.: Alaska simulator toolset for conducting controlled experiments on process flexibility. In: Soffer, P., Proper, E. (eds.) CAiSE Forum 2010. LNBIP, vol. 72, pp. 205-221. Springer, Heidelberg (2011)

42. Casati, F., Ceri, S., Pernici, B., Pozzi, G.: Workflow evolution. Data and Knowledge Engineering 24, 211-238 (1998)

43. Rinderle, S., Reichert, M., Dadam, P.: Evaluation of correctness criteria for dynamic workflow changes. In: van der Aalst, W.M.P., ter Hofstede, A.H.M., Weske, M. (eds.) BPM 2003. LNCS, vol. 2678, pp. 41-57. Springer, Heidelberg (2003)

44. Rinderle, S., Reichert, M., Dadam, P.: Correctness criteria for dynamic changes in workflow systems - a survey. Data and Knowledge Engineering 50, 9-34 (2004)

45. Weber, B., Reichert, M.: Refactoring process models in large process repositories. In: Bellahsène, Z., Léonard, M. (eds.) CAiSE 2008. LNCS, vol. 5074, pp. 124-139. Springer, Heidelberg (2008) 
46. Van der Aalst, W., Reijers, H., Weijters, A., van Dongen, B., de Medeiros, A.A., Song, M., Verbeek, H.: Business process mining: An industrial application. Information Systems 32, 713-732 (2007)

47. Günther, C., Rinderle, S., Reichert, M., van der Aalst, W.: Change mining in adaptive process management systems. In: Proc. CoopIS 2006, pp. 309-326 (2006)

48. Li, C., Reichert, M., Wombacher, A.: Discovering reference models by mining process variants using a heuristic approach. In: Dayal, U., Eder, J., Koehler, J., Reijers, H.A. (eds.) BPM 2009. LNCS, vol. 5701, pp. 344-362. Springer, Heidelberg (2009)

49. Li, C., Reichert, M., Wombacher, A.: The MinAdept clustering approach for discovering reference process models out of process variants. Int'l Journal of Coop. Inf. Sys. 19, 159-203 (2010) 